\title{
INFLUÊNCIA DA MELHORA NO PENEIRAMENTO DE SINTER NO PROCESO DOS ALTOS-FORNOS DA CSA*
}

\author{
André Wulff Hirano ${ }^{1}$ \\ Bruno Pinheiro da Silva ${ }^{2}$ \\ Geovane Viturino da Silva ${ }^{3}$ \\ Marilene Aparecida Ennes Landin ${ }^{4}$ \\ Maxwell Pereira Cangani ${ }^{5}$
}

\section{Resumo}

Um dos parâmetros relevantes no controle do fluxo gasoso em um alto-forno é o tratamento físico da matéria prima. Nos Altos-fornos da CSA através de ajustes em telas das peneiras de sínter elevou-se o rendimento de finos, trazendo benefícios significativos para o controle do fluxo gasoso. Neste trabalho é possível verificar a influência do aumento da eficiência de peneiramento na variação do fluxo gasoso e por consequência a redução no desvio padrão da carga térmica na cuba superior do Alto-forno. Com o fluxo gasoso mais estável, foi observado um aumento no rendimento de gás de topo e redução da variação na parede, assim foi observado os benefícios no fuel rate e produtividade dos Altos-fornos.

Palavras-chave: Sinter; Peneiramento; Alto-forno; Finos, Carga térmica.

\section{INFLUENCE OF IMPROVEMENT OF THE SINTER SCREENING IN CSA BLAST FURNACE PROCESS}

\section{Abstract}

One of the main parameters in the gas flow distribution in a Blast Furnace is the size distribution. In the CSA Blast Furnaces, the sinter fines extraction was improved through sieves adjustments in the stock house and a better gas flow control into the Blast Furnace was observed. In this paper is possible to evaluate the influence of the improvement in the sinter screening process in the BF gas flow and its impact in the reduction of thermal load standard deviation on the wall in the upper part of the Blast Furnace. By BF stable gas flow, it was observed a increasing in the top gas efficiency and a decreasing in the variation of the thermal load on the wall. Fuel rate and Hot Metal production was also improved as a consequence of better gas flow distribution into Blast Furnace.

Keywords: Sinter; Screening; Blast Furnace; Fines; Thermal load

1 Engenheiro Metalurgista, Coordenador de Unidade Técnica, CSA, Rio de Janeiro, RJ, Brasil.

2 Engenheiro Químico, Mestrando em Engenharia Metalúrgica e de Materiais, Engenheiro de Processo, CSA, Rio de Janeiro, RJ, Brasil.

3 Engenheiro Metalurgista, Mestre em Engenharia Metalúrgica, Engenheiro de Processo, CSA, Rio de Janeiro, RJ, Brasil.

4 Engenheira de Produção, Engenheira de Processo, CSA, Rio de Janeiro, RJ, Brasil.

5 Engenheiro de Materiais, Mestre em Engenharia de Materiais, Engenheiro de Processo, CSA, Rio de Janeiro, RJ, Brasil. 


\section{INTRODUÇÃO}

A operação estável de um Alto-forno está diretamente relacionada com a estabilidade do fluxo gasoso. Na CSA trabalhamos com a chamada "marcha central", onde o controle do gás é distribuído em sua maior parte pelo centro do alto forno, porém verificamos que o controle do fluxo periférico é tão importante quanto o fluxo no centro [1], e o bom equilíbrio da relação centro-parede é essencial, principalmente em elevada produtividade devido a deformação da zona de coesão. Assim observa-se que o controle de fluxo gasoso periférico tem impacto significativo tanto na estabilidade, quanto na vida útil dos staves dos Altos-fornos.

A refrigeração dos altos-fornos da CSA é feita por staves, que conta com um total de 8 fileiras de staves, as 4 fileiras na região da cuba superior são staves de ferro fundido e as outras 4 fileiras da região cuba inferior, ventre e rampa são de cobre. Eventos especiais como o carregamento de finos de sínter em excesso, podem causar perda da permeabilidade e, consequentemente, desvio do fluxo gasoso desde a zona granular até a zona de elaboração, trazendo prejuízos para estabilidade da carga térmica dos staves de ferro fundido e cobre, porém é muito perceptível os impactos diretos na cuba superior devido a variação da porosidade do leito.

O Carregamento de finos gera uma perda de permeabilidade localizada devido ao seu perfil de escoamento, desviando o fluxo gasoso que reduz o contato gás-carga, assim outros fenômenos começam a ocorrer como a perda de rendimento gasoso e elevação da redução direta. O resultado da variação da permeabilidade também é observado na perda térmica na região superior, tendo impactos direto na redução da produção por redução para proteção dos staves, e elevação no fuel rate para compensar a perda de rendimento e aumento das reações endotérmicas.

O objetivo deste trabalho é mostrar que uma adequada remoção dos finos traz benefícios significativos no processo de Alto-forno, com foco na variação da carga térmica dos staves de ferro fundido.

\section{MATERIAIS E MÉTODOS}

Um dos pilares no controle de processo dos Altos-fornos é o peneiramento dos materiais, e o sínter tem grande importância nessa etapa devido suas características e elevada participação na carga metálica, trazendo uma contribuição significativa na entrada de finos em um alto-forno.

Dessa forma os testes consistiram em substituir 3 fileiras de um total de 7 fileiras das duas peneiras de sínter, onde as malhas substituídas que eram de poliuretano com $5 \mathrm{~mm}$ e baixa área aberta por aço de $8 \mathrm{~mm}$ com suporte em poliuretano de elevada área aberta. Essa substituição gera dois principais impactos, um aumento do rendimento de finos, e uma redução no rendimento de grosso levando em consideração que a fração de corte objetivada é $5 \mathrm{~mm}$. Porém o impacto na estabilidade da carga térmica, redução do fuel rate e aumento de produtividade levaram a mais um passo no processo de evolução no controle de processo: melhora na eficiência de peneiramento de sínter, independente da configuração de malhas de peneiramento. 


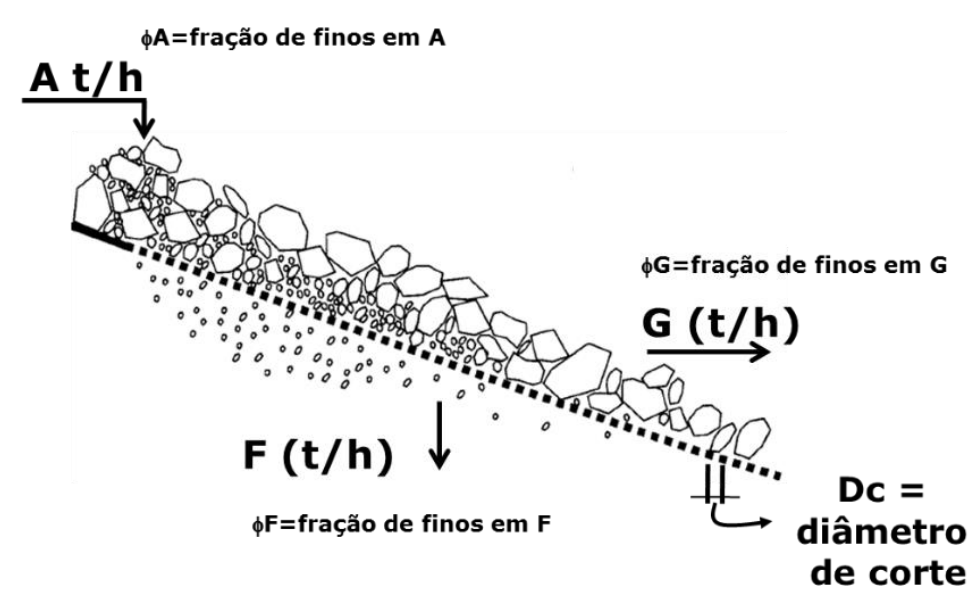

Figura 1. Rendimento de remoção de finos em uma peneira

Onde o rendimento de finos é dado pela expressão $\% \boldsymbol{\eta} \boldsymbol{F i n o s}=\frac{\emptyset \boldsymbol{F}}{\emptyset \boldsymbol{A}} \times \mathbf{1 0 0}$, onde para peneiras industriais espera-se um rendimento superior a $90 \%$.

\subsection{Escoamento gasoso no Alto-forno}

Para que as reações de redução ocorram, o gás deverá ter contato com carga trocando calor e massa. Tal que o leito deverá ter uma permeabilidade controlada, que depende fortemente das características das partículas e do fluido [2]. A figura 2 mostra a criação de vazios nas regiões inferiores do Alto-forno devido a troca de massa em um processo contra-corrente.

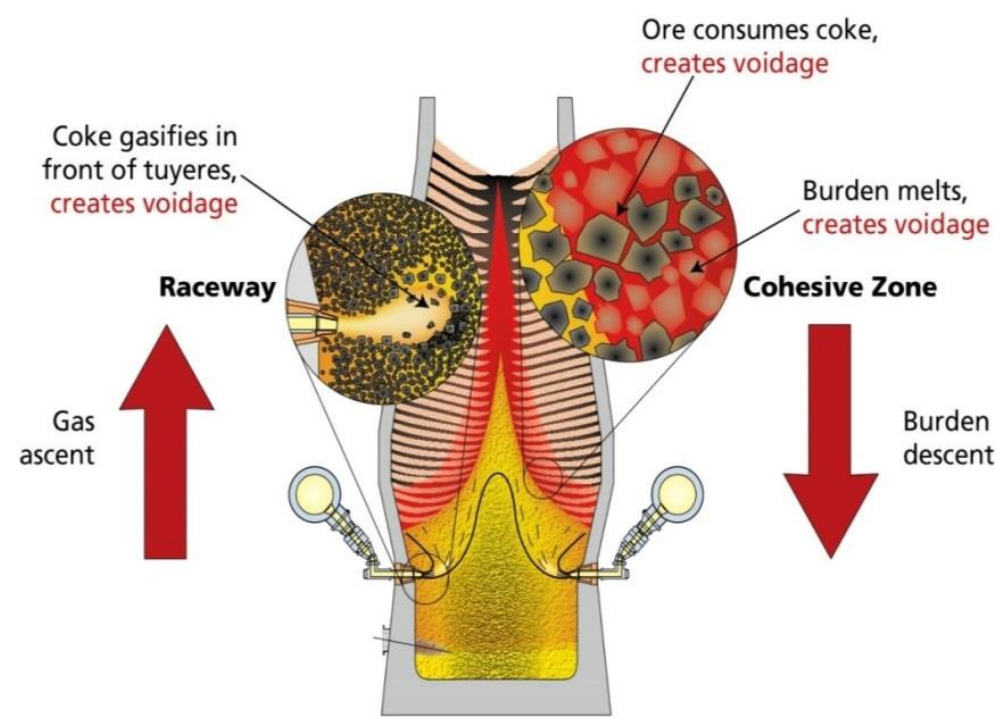

Figura 2. Criação de vazios regiões inferiores do Alto-forno [2] 
$\mathrm{Na}$ cuba superior a gaseificação não ocorre e a permeabilidade do fluxo gasoso depende fortemente da fração de vazios proveniente do empilhamento dos materiais, o qual depende da distribuição granulométrica das partículas em contato, a combinação desses fatores impacta diretamente no empacotamento do leito o qual aumenta quando a taxa de partículas finas aumenta chegando em seu mínimo em aproximadamente $25 \%$ de volume de finos no leito, como pode ser visto na figura 3 .

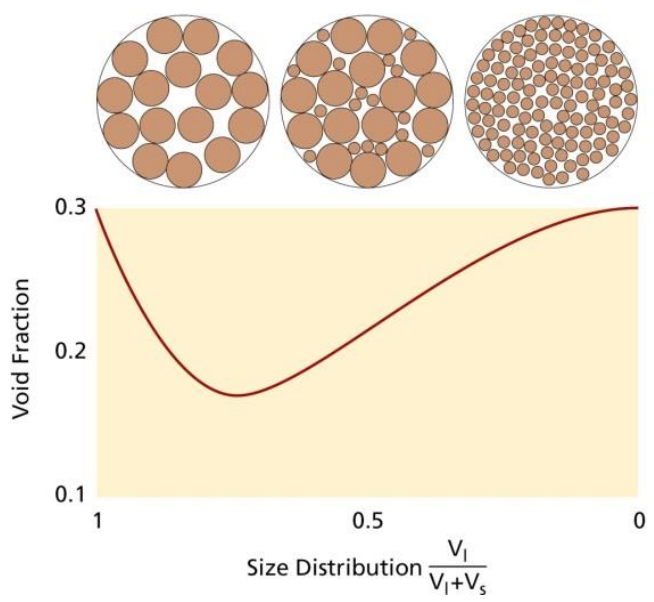

Figura 3. Geração de vazios em função da distribuição granulométrica [2]

A figura 3, retrata a existência de 2 tipos de partículas, uma grande $\left(V_{I}\right)$ e pequena $\left(V_{s}\right)$. O eixo $x$ fornece a relação a fração volumétrica de partículas grossas no volume total. Pode-se verificar que a dispersão granulométrica é de grande relevância na perda de porosidade no leito, que por sua vez prejudica a permeabilidade do fluxo gasoso [2]. À medida que o volume de partículas finas aumenta, elas preenchem os interstícios, causando essa diminuição da fração de vazios.

No caso do sínter, os fatores de forma e a degradação também impactam fortemente na formação dos espações vazios. Dependendo da sua participação no leito do forno e baseado nos fatores de forma, resistência e dispersão o sínter é de grande relevância no empacotamento da carga, e o controle dos finos de sínter carregados pode-se definir diretamente uma das propriedades da carga mais relevante para o processo, a porosidade do leito e sua variação.

\subsection{Deposição de finos no topo do Alto-forno}

ICHIDA et al. (1992) estudou a deposição de finos no topo do Alto-forno em função da distribuição da velocidade do gás de topo e do perfil da carga, em "V" ou "M". No formato "V" os finos tendem a se depositar na região central porque são lançados em uma região central. Já no formato "M", as partículas tendem a se depositar tanto na região central, como próximo da parede [3]. A figura 4 mostra a deposição dos finos em função do tipo de perfil de carga. 


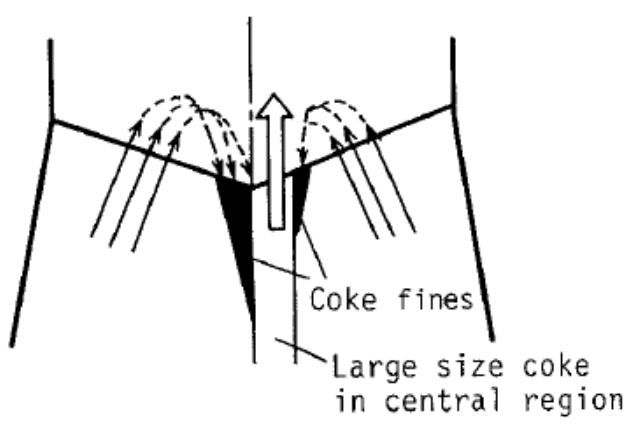

(1) V-shaped stock

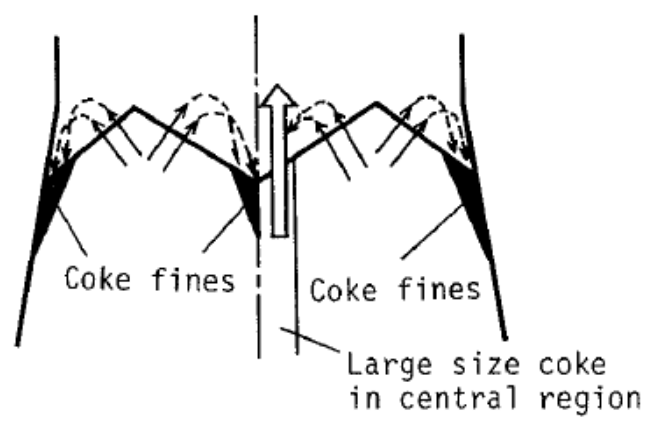

(2) M-shaped stock

Figura 4. Influência do perfil de carga e a distribuição granulométrica do coque na deposição de finos no topo do Alto-forno [3]

\subsection{Influência do carregamento de finos na carga térmica dos Altos-fornos}

A figura 5 mostra o resultado da carga térmica do Alto-forno 02 de Schwelgern após o carregamento de finos de coque. Verifica-se tanto uma elevação tanto do valor absoluto, quanto uma piora significativa no desvio da carga térmica na parte superior do forno.
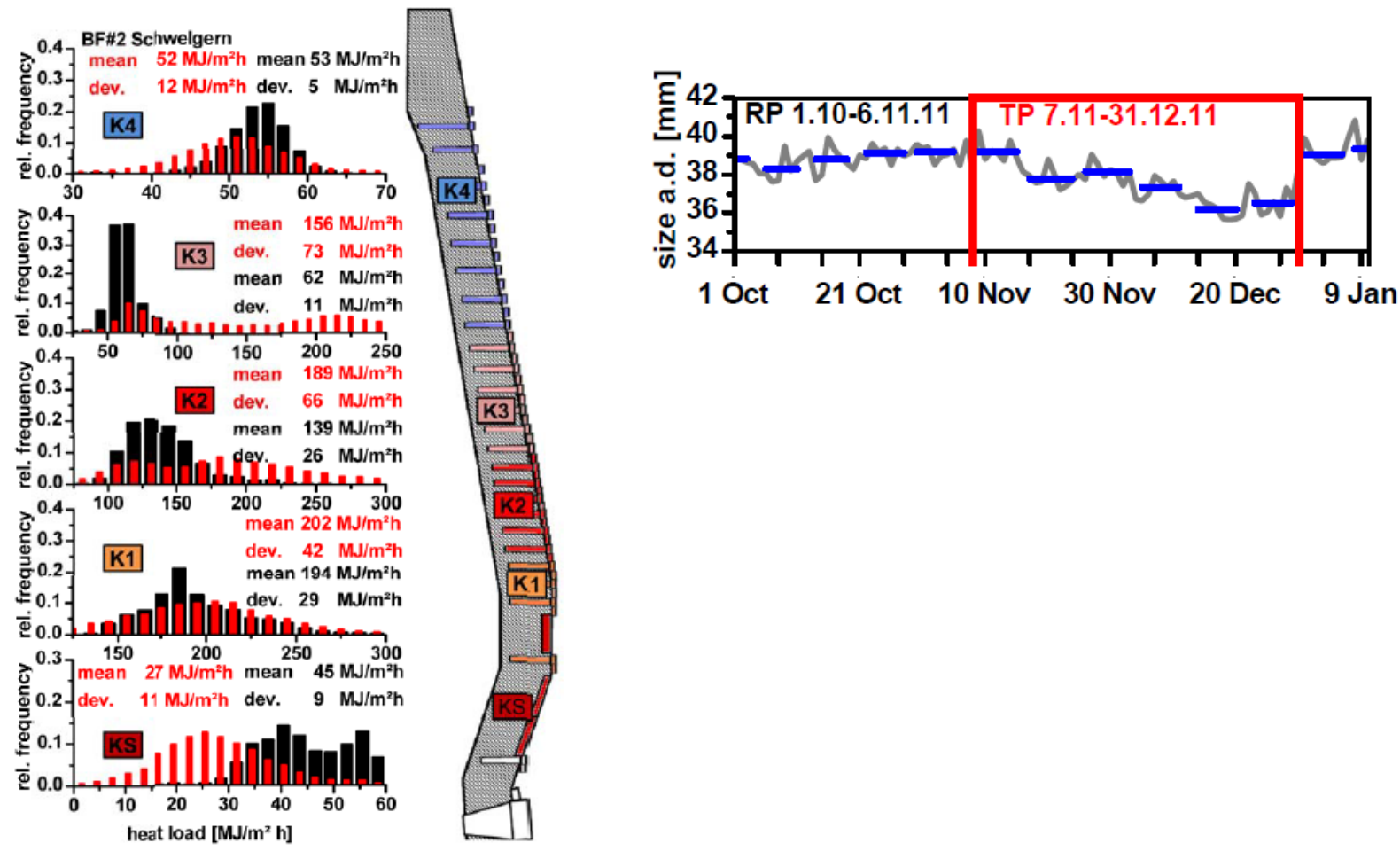

Figura 5. Carga térmica ao longo do Alto-forno 2 de Schwelgern após carregamento de finos de coque [4] 
Já na região da Rampa do Alto-forno, houve uma queda da carga térmica com elevação do desvio padrão. Segundo Klock et al. (2015), uma formação de cascão foi a causa deste fenômeno na região da rampa [4].

\section{RESULTADOS E DISCUSSÃO}

Visando melhorar a eficiência de peneiramento, foi utilizada 3 fileiras de telas de $8 \mathrm{~mm}$ no inicio do peneiramento (próximo à tela de impacto). A tabela 1 mostra 0 resultado no rendimento.

Tabela 1. Rendimentos de sínter antes e após inserção telas de 8mm nas peneiras de sínter

\begin{tabular}{|l|c|c|c|}
\hline $\begin{array}{c}\text { Peneira de } \\
\text { sinter }\end{array}$ & \multicolumn{1}{c}{$\begin{array}{c}\text { Rendimento de finos } \\
\text { modificação }\end{array}$} \\
\hline Undersize (F) & $\% \boldsymbol{\eta} \boldsymbol{F}=\frac{\boldsymbol{\phi} \boldsymbol{F}\left(\frac{\boldsymbol{t}}{\boldsymbol{h}}\right)}{\boldsymbol{\phi} \boldsymbol{F i n o s} \text { entrada }\left(\frac{\boldsymbol{t}}{\boldsymbol{h}}\right)} \times \mathbf{1 0 0}$ & $74 \%$ & $90 \%$ \\
\hline Oversize (G) & $\% \boldsymbol{\eta} \boldsymbol{G}=100-\% \boldsymbol{\eta} \boldsymbol{F}$ & $26 \%$ & $10 \%$ \\
\hline
\end{tabular}

Pode-se verificar uma melhora significativa na remoção de finos de sínter aumentando de $74 \%$ para $90 \%$ a eficiência de remoção de finos, porém impactos negativos no retorno de material entre 5 e $8 \mathrm{~mm}$ para a sinterização foram observados.

O impacto positivo no Alto-forno leva à busca por uma melhora no sistema de peneiramento, independente da configuração de telas e dos métodos de controle do peneiramento, ele deve ser otimizado a atender o melhor cenário econômico, assim deve ser levado em consideração os impactos na sinterização. Desta forma, novos testes serão realizados visando otimizar a extração de finos minimizando os impactos na sinterização, e mantendo a estabilidade operacional do alto-forno.

\subsection{Impacto da melhora da eficiência de peneiramento no processo de Alto- forno}

Em termos de carga térmica, pode-se verificar após a alteração no sistema de peneiramento o aumento da eficiência levou a um menor carregamento de finos de sínter, que teoricamente levaria a uma menor variação da permeabilidade e menor desvio de fluxo gasoso [2], onde esse fato pode ser evidenciado pelo menor desvio padrão da carga térmica. A figura 6 mostra o desvio padrão da carga térmica dos staves de ferro fundido, onde observa-se uma menor variação das amplitudes na carta de controle devido a maior estabilidade da parede, já a figura 7 evidencia que além da redução média de $216 \mathrm{kw}$ no desvio padrão carga térmica, o comportamento entres os dias é significativamente diferente e ao levarem o valor de $P$ para zero nos testes de Bonett e Levene indicando uma maior estabilidade entre os dias. 


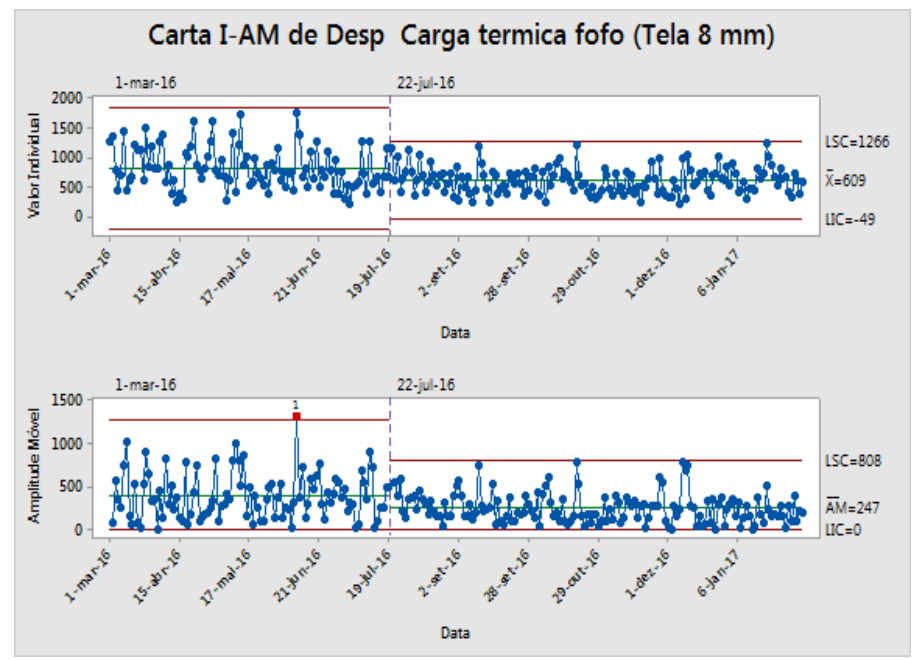

Figura 6. Desvio padrão da carga térmica dos staves de ferro fundido (cuba superior).

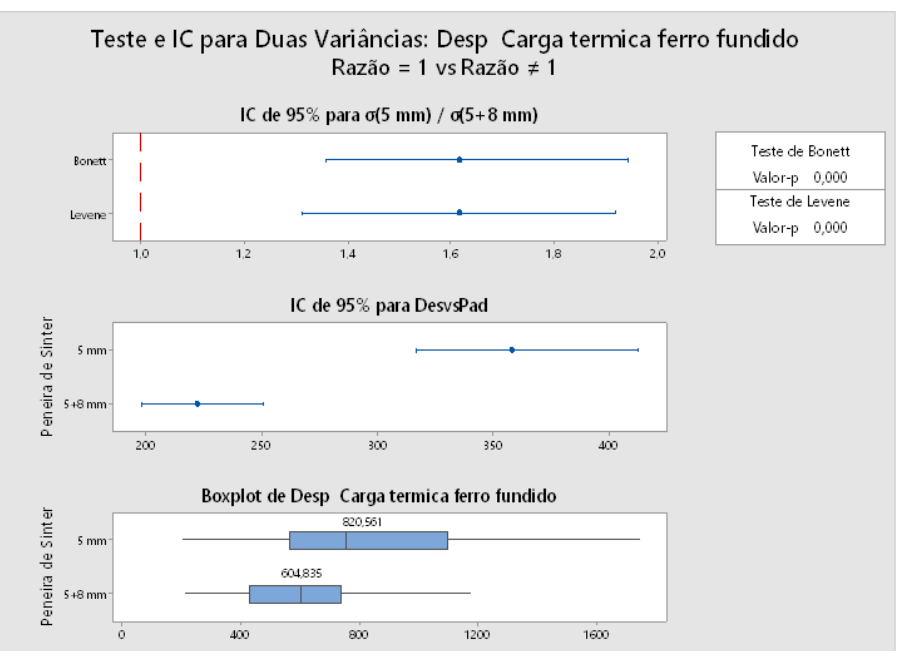

Figura 7. 2-sample t teste para duas variâncias para a carga térmica no ferro fundido.

Com o gás mais estável (desvio padrão da carga térmica menor), nota-se um aumento do rendimento do gás no topo. A figura 8 mostra a evolução do rendimento gasoso em função do desvio padrão da carga térmica dos staves de ferro fundido, sendo o desvio padrão da carga térmica dos staves de ferro fundido categorizados da seguinte maneira: $A<487 \mathrm{kw}<\mathrm{B}<677 \mathrm{kw}<\mathrm{C}<897 \mathrm{kw}<\mathrm{D}$, ou seja, à medida em que 0 desvio padrão da carga térmica é reduzido o rendimento do gás no topo aumenta. 


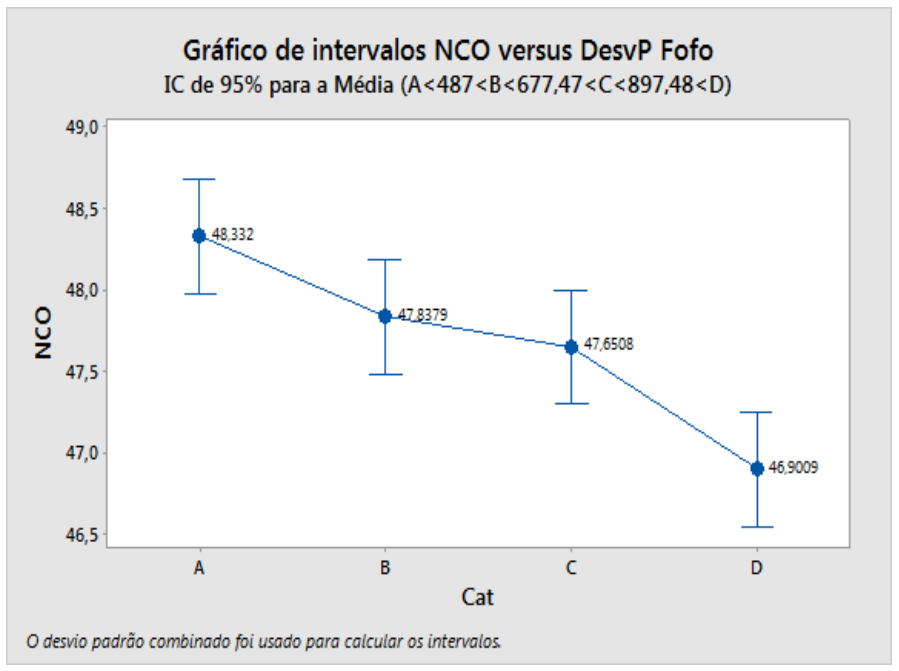

Figura 8. Rendimento do gás no topo do desvio padrão da carga térmica dos staves de ferro fundido.

Foi possível aproximar o efeito positivo no rendimento para a seguinte relação linear, $\mathrm{NCO}=48,81-0,001583^{\star}$ DesvPFofo. Levando em consideração a redução média 216 kw no desvio padrão da carga térmica no Fofo, chegamos em um aumento médio de $0,34 \%$ no rendimento de gás.

O fuel rate do Alto-forno sofreu os efeitos da maior estabilidade, conforme pode-se verificar na figura 9 , a qual utiliza as mesmas faixas da figura 8 $(\mathrm{A}<487 \mathrm{kw}<\mathrm{B}<677 \mathrm{kw}<\mathrm{C}<897 \mathrm{kw}<\mathrm{D})$.

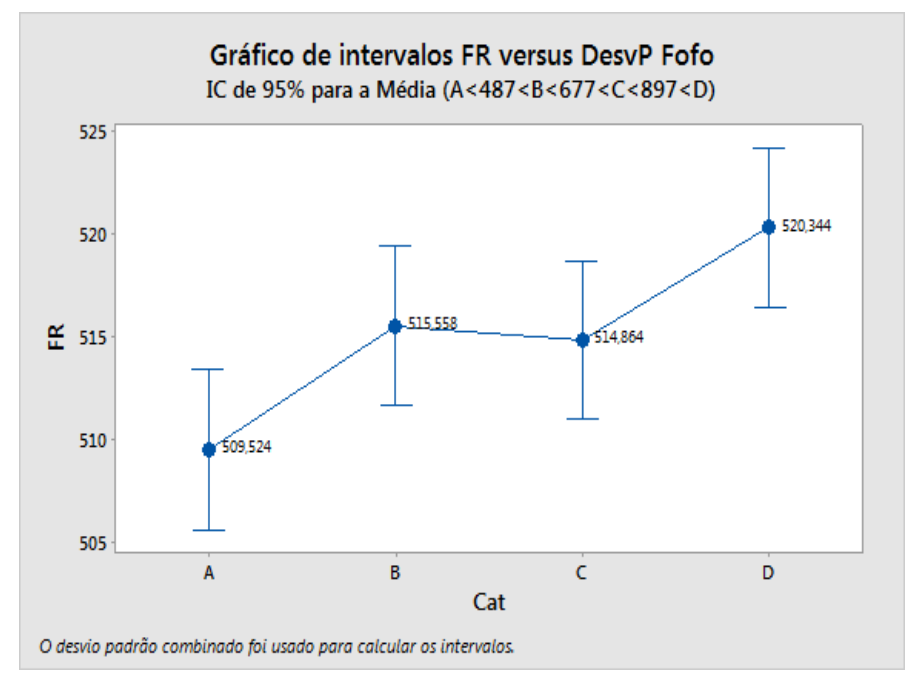

Figura 9. Fuel rate em função do desvio padrão da carga térmica dos staves de ferro fundido.

Finalmente, pode-se observar devido ao aumento da estabilidade uma melhora na produtividade do Alto-forno em função do melhor peneiramento, conforme mostra a figura 10 a produtividade média no cenário $A$ antes da troca da malha era de 2,2 $\mathrm{t} / \mathrm{m}^{3} /$ dia e após a troca da malha no cenário $\mathrm{B}$ a produtividade média foi para 2,33 $\mathrm{t} / \mathrm{m}^{3} /$ dia. Normalmente, quando há o aumento do desvio padrão da carga térmica, necessita-se uma redução do volume de gás através da vazão de sopro para a normalização da marcha e aumento do fuel rate para compensar as perdas térmicas, levando diretamente a uma redução da produção. 


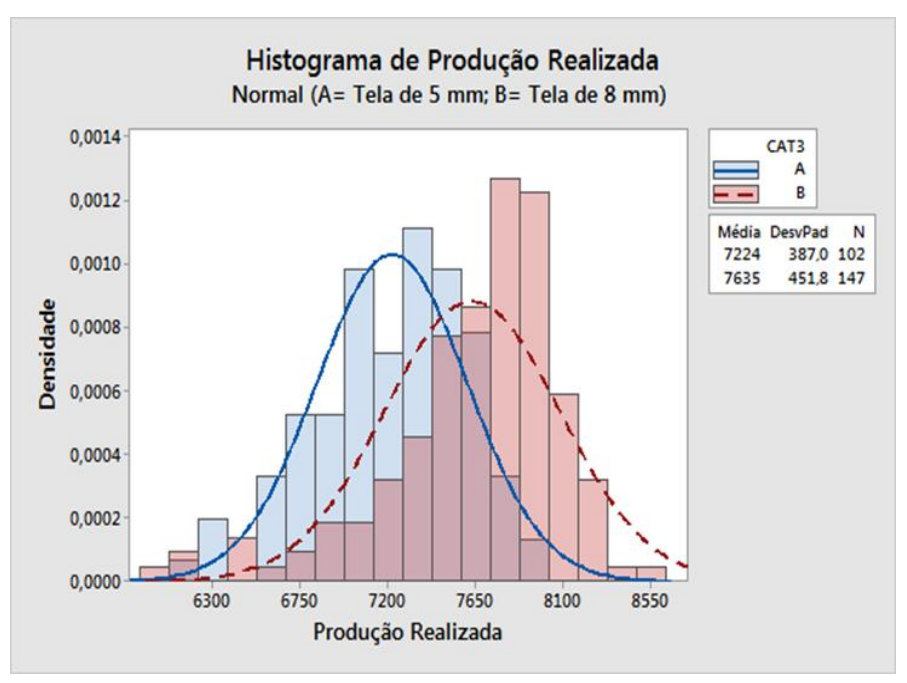

Figura 10. Melhora na produtividade do Alto-forno após utilização das telas de 8mm

\section{CONCLUSÃO}

Controle da carga térmica dos Altos-fornos da CSA é um dos parâmetros dos mais relevantes para manutenção da estabilidade, pois o equilíbrio do forno e do controle térmico depende dessa estabilidade. Um aumento no desvio padrão prejudica diretamente esse equilíbrio e significando uma maior perda de energia do processo, seja pelo aumento da carga térmica, pela redução do contato gás-carga gerando uma queda no rendimento ou pelo aumento das reações de redução direta devido a maior chegada de material mal elaborado na zona de coesão.

Dessa forma pode-se observar que apesar da piora no rendimento de grosso, a melhora do peneiramento de sínter mostrou forte influência no processo do Altoforno, notadamente no desvio padrão da carga térmica dos staves de ferro fundido gerando efeitos positivos como:

- Aumento do rendimento do gás no forno

- Maior estabilidade e aproveitamento do gás permitindo a redução do fuel rate.

- Maior estabilidade permitindo um aumento da produtividade. 


\section{REFERÊNCIAS}

1. Silva, B; Hirano, A; Torres, E; Ramos, V. Evolução do controle operacional do Alto Forno 1 da TKCSA, ABM, 2014, Minas Gerais, Brasil.

2. GEERDES, M.; et al. Modern Blast Furnace Ironmaking: An introduction, $3^{\underline{a}}$ ed., 2015.

3. ICHIDA, M.; et al. Behavior of fines in the Blast furnace. ISIJ international, Vol. 32, 1992, № 4, pp. 505-513.

4. Schwalbe, R.; Klock, R.; Janhsen, U.; Schmöle, P.; Peters, M. Influence of intentionally deteriorated coke properties on performance of Blast Furnace \#2 Schwelgern, METEC \& $2^{\text {nd }}$ ESTAD, 2015, Dusseldorf, Alemanha. 